\title{
Gimnasio de simulación quirúrgica: una herramienta educacional factible de entrenamiento continuo. Experiencia de un centro universitario
}

\author{
Surgical simulation gym: a feasible educational tool for continuous training. \\ Experience of a university center
}

Cristián Jarry,*,** Martín Inzunza,*,** José Quezada,*,*** Carlo Marino,*,***

Elga Zamorano,**** Valeria Alvarado,***** Carlos Martínez,*****

Eduardo Machuca, ${ }^{* * * * *}$ Pablo Achurra, $* * * * * *$ Julián Varas*******

Palabras clave: Simulación, laparoscopia, gimnasio.

Key words: Simulation, laparoscopy, gymnasium.

* Becario de investigación, Centro de Simulación y Cirugía Experimental. ** Residente de Cirugía General, División de Cirugía, Facultad de Medicina.

*** Residente de Cirugía Digestiva, División de Cirugía, Facultad de Medicina.

**** Enfermera

Coordinadora, Centro

de Simulación y Cirugía Experimental.

***** Médico Veterinario, instructor de Programas de Laparoscopia, Centro de Simulación y Cirugía Experimental.

****** Cirujano Digestivo, División de Cirugía, Facultad de Medicina. ******* Coordinador Médico, Centro de Simulación y Cirugía Experimental.

Pontificia Universidad Católica de Chile.

Recibido: 20/02/2019 Aceptado: 15/03/2019

\section{RESUMEN}

\section{ABSTRACT}

Introducción: Adquirir competencias en cirugía laparoscópica requiere completar largas curvas de aprendizaje que son susceptibles de acortarse mediante el entrenamiento simulado. Sin embargo, las instancias de formación de este tipo suelen ser breves, esporádicas y poco costo-efectivas. Como ocurre en otras disciplinas, contar con un formato de entrenamiento continuo a modo de "gimnasio" permitiría mejorar la adquisición de habilidades. Objetivos: Describir la implementación de un gimnasio quirúrgico donde realizar programas de aprendizaje laparoscópico dirigidos a profesionales laparoscopistas. Material y métodos: Estudio descriptivo. Se implementó un espacio físico donde realizar entrenamiento tutorizado por instructores expertos en educación médica y laparoscopia. Se ofrecieron a los alumnos cuatro cursos de simulación para el desarrollo de habilidades básicas y avanzadas de laparoscopia y la posibilidad de asistir a entrenar bajo su propia disponibilidad de tiempo. Resultados: En siete años se han formado 435 personas en laparoscopia, todas ellas en laparoscopia básica, 240 en laparoscopia avanzada, 62 en el módulo de laparoscopia esofagogástrica y bariátrica (EGB) y 11 en laparoscopia hepatobiliar y pancreática (HBP). La evaluación por parte de los asistentes externos como residentes es positiva. Conclusiones: El establecimiento de un gimnasio de simulación para el desarrollo y mantenimiento de habilidades quirúrgicas es una opción factible y atractiva para residentes y cirujanos.
Introduction: Acquiring competences in laparoscopic surgery requires completing long learning curves that can be effectively shortened through simulated training. However, the training instances of this type are usually brief, sporadic and non cost-effective. As in other disciplines, having a continuous training format as a "gymnasium" would improve the acquisition of skills. Objectives: To describe the implementation of a surgical gymnasium where laparoscopy professionals can learn through validated training programs. Material and methods: Descriptive study. Infrastructure and a physical space were implemented to conduct training tutored by expert instructors in medical education and laparoscopy. Four simulation courses were offered to students for the development of basic and advanced laparoscopic skills. All students had the possibility of attending the gymnasium under their own time availability. Results: In seven years, 435 people have been trained in laparoscopy, all of them in basic laparoscopy, 240 of them in advanced laparoscopy, 62 in the esophagogastric and bariatric laparoscopy module and 11 in hepatobiliary and pancreatic laparoscopy. The evaluation made both by external assistants and residents is positive. Conclusions: The establishment of a simulation gym for the development and maintenance of surgical skills is a feasible and attractive option for residents and surgeons.

\section{INTRODUCCIÓN}

T a cirugía ha cambiado de manera sigـnificativa durante los últimos años. Sin importar el grado de complejidad, la mayoría de los procedimientos abdominales hoy en día se realiza por vía laparoscópica o mínimamente invasiva. ${ }^{1}$ No obstante, la curva de aprendizaje de la cirugía por laparoscopia es difícil y lenta. Lo anterior, sumado a demandas de mayor seguridad por parte de los sistemas de salud, ha cambiado también la forma de 
cómo se enseñan y entrenan las habilidades quirúrgicas. ${ }^{1-4}$

En este contexto, la simulación ha adquirido un rol fundamental como herramienta educacional complementaria para la adquisición de habilidades técnicas. Se han desarrollado múltiples módulos de entrenamiento que en la actualidad forman parte del currículo mínimo para la especialización. ${ }^{3,5,6}$ Muchos de estos módulos son básicos y realizados de forma acotada, y en ocasiones, son complementados con cursos prácticos o "hands-on" de tiempo limitado $^{7}$ (habitualmente, inferiores a tres días). Para aprender habilidades más avanzadas, la alternativa es entrenarse en cursos abreviados que utilizan animales vivos anestesiados o cadáveres humanos, opciones de alto costo y no siempre disponibles. Bajo este paradigma, las curvas de aprendizaje para la obtención de habilidad técnica para procedimientos complejos en pacientes son difíciles de alcanzar y muy dependientes de la casuística local. De esa forma, el cirujano se entrena sobre todo en el escenario quirúrgico, con los costos y riesgos implicados. ${ }^{8-10}$

Disciplinas como la música o el deporte de alto rendimiento también requieren incorporar habilidades específicas de gran carga cognitiva para ser evaluadas en ambientes de estrés y con expectativas de alto desempeño. ${ }^{11}$ Tanto músicos como deportistas cumplen estrictos horarios de entrenamiento a lo largo de toda su vida profesional. ${ }^{12}$ El escenario, concierto o campeonato representa para ellos una mínima fracción de su actividad, donde demuestran las destrezas previamente adquiridas en un ambiente protegido.

Si bien la actividad quirúrgica comparte similitudes evidentes con el desarrollo de los oficios descritos, parecería que el paradigma de formación está invertido: el tiempo de entrenamiento resulta escaso para el cirujano, y como consecuencia, un porcentaje importante del proceso de aprendizaje se realiza en pabellón, donde hay limitado tiempo de retroalimentación ${ }^{13}$ y poco margen de error. El paradigma común de un gimnasio de entrenamiento presenta ventajas que a la fecha no se han podido replicar en la docencia quirúrgica. En éstos, el tiempo de entrenamiento es individualizado, se permite y promociona la asistencia constante a lo largo del año, existen profesionales capacitados en evaluar el desempeño y plantear nuevos desafíos o metas.

En el presente texto describiremos un modelo exitoso de entrenamiento quirúrgico basado en los principios de práctica deliberada y retroalimentación dirigida, desarrollado en un espacio accesible y disponible bajo la modalidad que hemos denominado "gimnasio".

\section{MATERIAL Y MÉTODOS}

Estudio descriptivo en el cual se presentará la experiencia de un centro de simulación universitario. Los datos fueron obtenidos de la documentación propia del centro y de publicaciones asociadas.

\section{Objetivo}

Describir la implementación de un gimnasio quirúrgico donde realizar programas de aprendizaje laparoscópico basados en evidencia, dirigidos a profesionales para quienes la laparoscopia resulta una herramienta útil en su quehacer.

\section{Población objetivo}

Residentes de cirugía, urología o ginecología de primer o segundo año, además de especialistas del área de la cirugía, urología, ginecología y medicina veterinaria con interés en el desarrollo de habilidades quirúrgicas laparoscópicas básicas y avanzadas.

\section{Espacio físico y herramientas de aprendizaje}

Para la implementación de este modelo, se dispuso del Centro de Simulación de la Pontificia Universidad Católica de Chile (PUC), ubicado en un edificio contiguo al Hospital Clínico de la Universidad Católica, donde los residentes de especialidades quirúrgicas realizan como mínimo 50\% de sus rotaciones clínicas. El centro cuenta con 480 metros cuadrados; de ellos, aproximadamente 80 son de uso exclusivo para el aprendizaje laparoscópico.

El entrenamiento se realizó en uno de los 12 simuladores laparoscópicos desarrollados 
por nuestro equipo. ${ }^{14}$ Se utilizaron modelos sintéticos para el módulo básico y tejido ex vivo bovino y porcino para el entrenamiento de módulos avanzados.

\section{Recurso humano}

Se contó con profesionales de dedicación exclusiva involucrados en el desarrollo de estos cursos. Dos cirujanos, además de tres veterinarios con experiencia en cirugía laparoscópica, cumplieron el rol de docentes. Este equipo fue previamente formado en laparoscopia, simulación y herramientas de educación médica como la correcta entrega de retroalimentación efectiva. Además, dos técnicos fueron asignados para la preparación del tejido ex vivo y mantenimiento del equipamiento. La coordinación horaria y gestión de los cursos fue realizada por personal administrativo del centro (una enfermera universitaria y una secretaria).

\section{Cursos impartidos}

Se realizaron cuatro cursos bajo modalidad gimnasio (Tabla 1). Éstos fueron elaborados bajo una lógica constructivista de aprendizaje e impartidos por docentes expertos en laparoscopia y docencia quirúrgica. ${ }^{15}$ Se validó cada curso en equivalencia de horas académicas acorde a las sesiones determinadas, configurándose como diplomados certificados por la universidad. Se establecieron criterios de aprobación, reprobación y un plazo máximo de realización de cuatro meses para cada curso. Una vez realizada la inversión inicial en infraestructura y simuladores, los cursos se sostienen con base en costos operativos de entre 367 y 1,398 USD, considerando materiales, instrumental y docencia personalizada.

\section{Laparoscopia básica}

Módulo de desarrollo de habilidades básicas; consiste en 12 estaciones, con una equivalencia de nueve horas y cinco créditos (unidad de avance curricular local) (Figura 1).

\section{Laparoscopia avanzada}

Módulo orientado a desarrollar la habilidad de sutura intracorpórea, con el objetivo de realizar anastomosis intestinal laterolateral en modelo ex vivo. Se estableció como prerrequisito la aprobación del módulo básico y se organizó en 16 sesiones de dificultad progresiva, equi-

Tabla 1. Módulos de entrenamiento.

\begin{tabular}{|c|c|c|c|c|}
\hline Curso & $\begin{array}{l}\text { Sesiones (horas } \\
\text { de equivalencia } \\
\quad \text { curricular) }\end{array}$ & Objetivos & Prerrequisitos & $\begin{array}{c}\text { Costo } \\
\text { aproximado } \\
\text { (al 2018, USD) }\end{array}$ \\
\hline $\begin{array}{l}\text { Entrenamiento de habilidades en } \\
\text { laparoscopia, nivel básico }\end{array}$ & $12(90)$ & $\begin{array}{l}\text { Entrenamiento en habilidades } \\
\text { básicas de laparoscopia }\end{array}$ & Ninguno & 367 \\
\hline $\begin{array}{l}\text { Entrenamiento de habilidades en } \\
\text { laparoscopia, nivel avanzado }\end{array}$ & $16(90)$ & $\begin{array}{l}\text { Entrenamiento en sutura } \\
\text { intracorpórea y anastomosis } \\
\text { intestinal manual }\end{array}$ & $\begin{array}{l}\text { Módulo } \\
\text { básico }\end{array}$ & 495 \\
\hline $\begin{array}{l}\text { Entrenamiento en cirugía laparoscópica } \\
\text { esofagogástrica y bariátrica }\end{array}$ & $24(180)$ & $\begin{array}{l}\text { Entrenamiento en } \\
\text { gastroyeyunoanastomosis y } \\
\text { anastomosis intestinal } \\
\text { Entrenamiento en } \\
\text { procedimiento antirreflujo }\end{array}$ & $\begin{array}{l}\text { Módulo } \\
\text { avanzado }\end{array}$ & 1,308 \\
\hline $\begin{array}{l}\text { Entrenamiento en cirugía laparoscópica } \\
\text { del hígado, vía biliar y páncreas }\end{array}$ & $23(180)$ & $\begin{array}{l}\text { Entrenamiento en } \\
\text { pancreatoyeyunoanastomosis } \\
\text { Entrenamiento en } \\
\text { coledocoyeyunoanastomosis }\end{array}$ & $\begin{array}{l}\text { Módulo } \\
\text { avanzado }\end{array}$ & 1,165 \\
\hline
\end{tabular}


valentes a un total de cinco créditos (90 horas) (Figura 2). La utilidad y transferencia de este módulo ha sido reportada en la literatura. ${ }^{16}$

\section{Laparoscopia esofagogástrica y bariátrica (EGB)}

Módulo dedicado a cirujanos generales y laparoscopistas de otras áreas, cuyo eje de aprendizaje se centra en cirugía digestiva de esófago, estómago y bariátrica. Se estableció como prerrequisito la aprobación de los módulos básico y avanzado y se organizó en 24 sesiones, entre las cuales destaca la realización de hernioplastia hiatal y funduplicatura de Nissen en modelo simulado ex vivo, además de la profundización en la habilidad de anastomosis

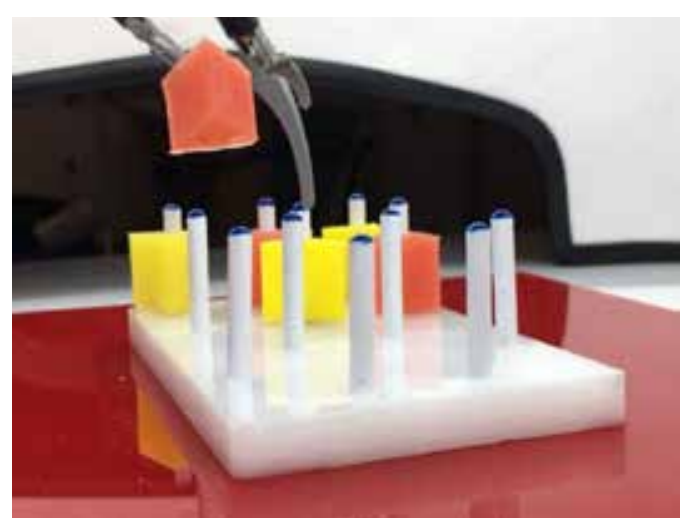

Figura 1. Visión interna del simulador laparoscópico en la sesión de módulo básico.

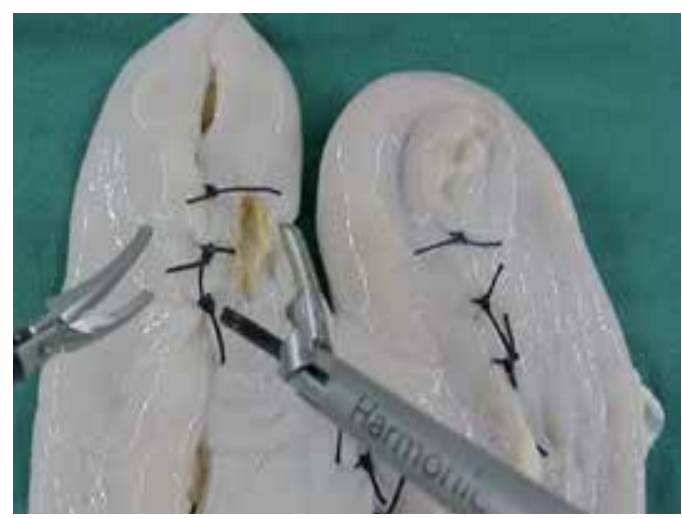

Figura 2. Visión del alumno en la sesión de módulo avanzado de habilidades en laparoscopia, donde debe realizar anastomosis laterolateral manual. intestinal. Su equivalencia fue de 180 horas cronológicas (10 créditos) (Figura 3).

\section{Laparoscopia hepatobiliar y pancreática $(\mathrm{HBP})$}

Módulo dedicado a cirujanos generales y laparoscopistas de otras áreas, con énfasis en cirugía hepatobiliar y pancreática. Se estableció como prerrequisito la aprobación de los módulos básico y avanzado y se organizó en 23 sesiones, entre las cuales destaca la realización de coledocoyeyunoanastomosis y pancreatoyeyunoanastomosis. Su equivalencia fue de 180 horas cronológicas (10 créditos) (Figura 4).

\section{Modalidad gimnasio}

Se describe a continuación el funcionamiento de la modalidad gimnasio. Sus pilares son los mismos que los de los gimnasios deportivos: un espacio de entrenamiento de disponibilidad plena, con personal capacitado en guiar el proceso de aprendizaje y un alumno motivado y responsable.

El proceso de aprendizaje comienza con la inscripción del alumno en alguno de los cursos. Luego de ello, recibe material de estudio con las bases teóricas y tutoriales en video de cada entrenamiento. De manera simultánea, debe agendar (vía correo electrónico o teléfono) sus sesiones de entrenamiento según disponibilidad. La oferta horaria del centro es de lunes a viernes de 8 a 19 horas durante todo el año, con capacidad para albergar 12 alumnos de manera simultánea. Los docentes y técnicos se anticipan a cada sesión, preparando lo necesario.

El laparoscopista en formación es instruido en la preparación del modelo o escenario simulado previamente a cada etapa, fomentando la autonomía. Una vez listo, es asignado al simulador reservado y comienza una sesión con objetivos preestablecidos. Cada sesión tarda entre una y tres horas; no se recomiendan sesiones de mayor tiempo. Además de sesiones específicas donde hay retroalimentación obligatoria, el alumno puede solicitar retroalimentación cuando estime necesario. Al finalizar, debe firmar su asistencia. La evaluación se realiza a 


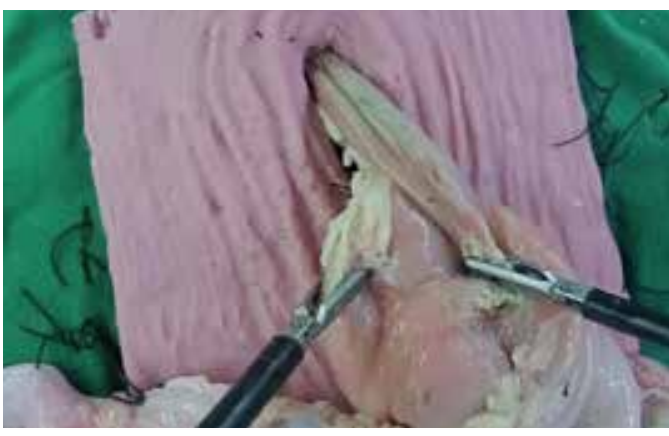

Figura 3. Visión del alumno en una sesión del módulo EGB, donde debe realizar una funduplicatura de Nissen.

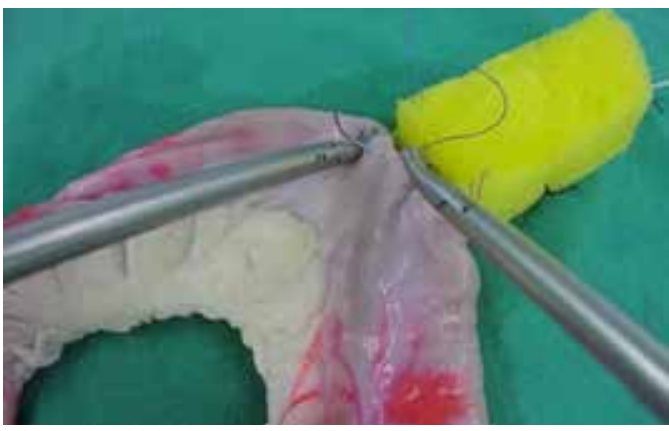

Figura 4. Visión del alumno en una sesión del módulo HBP, donde debe realizar pancreatoyeyunoanastomosis.

demanda en el módulo básico y en sesiones determinadas en los cursos avanzados, EGB y HBP. La certificación a nivel de diplomado se obtiene con la aprobación de tres cursos.

\section{RESULTADOS}

\section{A nivel de centro}

En un plazo de siete años, nuestro centro ha desarrollado los cuatro cursos mencionados bajo los principios y modalidad descrita. Hasta junio de 2018, nuestro centro ha formado a un total de 435 personas en laparoscopia, todas ellas en laparoscopia básica, 240 en laparoscopia avanzada, 62 en el módulo EGB y 11 en HBP. La evaluación por parte de los asistentes es, en general, positiva, lo cual se ha objetivado mediante una encuesta que evalúa cada uno de los aspectos del curso. Del total de evaluaciones positivas posibles de obtener en la encuesta, los módulos básico y avanzado han logrado 97.8 y $98.8 \%$, respectiva- mente, considerando 126 y 29 alumnos que han completado los módulos sólo entre 2017 y 2018.

\section{A nivel universitario}

La residencia de cirugía general incorporó en el año 2010 los módulos básico y avanzado como parte del currículo mínimo. Hoy, los residentes cuentan con tiempo protegido para entrenarse en modalidad gimnasio durante rotaciones de menor demanda asistencial. Se necesitan datos locales para evaluar el costo-beneficio de la implementación de esta medida en la residencia; sin embargo, a siete años de funcionamiento, la aceptación en general es positiva y se ha logrado formar más de 60 residentes PUC en laparoscopia avanzada (datos en proceso de publicación). Esta cifra considera, además, las residencias de ginecología y urología, que también han comenzado a exigir ambos módulos (básico y avanzado) como parte del currículo mínimo.

Por otro lado, la existencia de un gimnasio quirúrgico ha abierto la posibilidad de introducir a alumnos de pregrado en laparoscopia y, a la vez, ha sido una herramienta de gran utilidad de investigación en simulación quirúrgica.

\section{A nivel de extensión}

La modalidad gimnasio ha permitido entregar oportunidades formativas más allá de los módulos descritos. Con frecuencia, cirujanos o residentes acuden a realizar calentamiento previo a cirugías de mayor complejidad. Además, la modalidad de funcionamiento ha sido replicada por nuestro equipo en congresos y jornadas de sociedades quirúrgicas, con buena aceptación por parte de quienes han tenido la experiencia de gimnasio. ${ }^{17}$

Por último, a nivel nacional, el gimnasio quirúrgico actualmente se ha convertido en un espacio de desarrollo para herramientas de acreditación quirúrgica, como ocurre en el área de trauma, y se ha ampliado la modalidad a otras áreas como microcirugía y laparoscopia neonatal (datos en proceso de publicación).

\section{DISCUSIÓN}

Las instancias de entrenamiento cumplen un rol fundamental a la hora de adquirir cualquier 
competencia técnica. La práctica deliberada y sostenida en el tiempo en disciplinas como el deporte de alto rendimiento o la música son requisitos para un desempeño adecuado. El entrenamiento quirúrgico parece realizarse principalmente en la sala operatoria, donde la oportunidad de errar y recibir retroalimentación se ve limitada por el contexto.

La simulación ha ido cobrando progresiva relevancia en la educación médica y quirúrgica; sin embargo, los módulos de entrenamiento certificados son escasos y sin una propuesta de continuidad. La mayoría duran menos de cinco días una vez al año, y tal como ocurre en un gimnasio donde se levantan pesas, entrenamientos breves y esporádicos no permiten asegurar resultados favorables a nivel educacional ni psicomotor. De esa manera, se pone en riesgo el mantenimiento de las habilidades adquiridas, dado que la sostenibilidad de la curva de aprendizaje se hace por completo dependiente de la casuística personal. Esto resulta inaceptable en centros de bajo volumen, o bien cuando se requiere competencia ante procedimientos infrecuentes y/o complejos. Un centro donde entrenar a demanda las habilidades necesarias para un buen desempeño clínico parece una solución atractiva a este problema.

Ha sido presentada una experiencia de gimnasio quirúrgico de más de siete años de trayectoria. Los pilares fundamentales son la existencia de un lugar con amplia disponibilidad horaria, la presencia de docentes expertos y alumnos motivados.

Proponemos un centro de simulación donde colaboran diferentes profesionales (veterinarios, técnicos, residentes, cirujanos, enfermeras, secretarias) con la finalidad exclusiva de mantener un lugar con amplia disponibilidad horaria donde recibir un entrenamiento de calidad. En cuanto a logística, destacamos como sistemas claves el diálogo entre la calendarización personal de actividades y el equipo docente, la cadena de obtención y preparación del material ex vivo y la cercanía del espacio físico al centro docente-asistencial.

En segundo lugar, proponemos un equipo docente experto, de dedicación exclusiva a la enseñanza quirúrgica. Además, reconocemos como fundamental la existencia de programas estructurados de enseñanza, elaborados bajo un modelo constructivista y con respaldo en la literatura. Destacamos como principal herramienta pedagógica la retroalimentación efectiva; por ello, nuestros docentes son expertos no sólo en las habilidades técnicas, sino, además, en identificar errores frecuentes y comunicar con claridad sus soluciones.

Respecto a la motivación de aprendizaje, creemos que un programa estructurado y a la vez flexible resulta especialmente atractivo para profesionales que cumplen una alta demanda horaria, como ocurre con residentes y especialistas. Además, la adquisición progresiva de habilidades y el cumplimiento de metas con potencial de transferencia a la clínica es, sin duda, un elemento motivante.

La optimización del tiempo docente es una oportunidad de mejora, ya que la presencia de tiempo completo de tres docentes tiene un costo elevado. Se ha propuesto la docencia vía una aplicación de teléfono móvil, con retroalimentación personalizada no presencial (datos locales en proceso de publicación). De esta manera, el rol docente podría compatibilizarse con la práctica clínica, pudiendo el docente experto guiar el proceso de aprendizaje desde su hogar o el hospital.

Si bien se ha reportado la transferencia efectiva de habilidades entrenadas en simulación al escenario quirúrgico, carecemos de suficientes estudios que describan el impacto de esto sobre resultados clínicos. A nuestro conocer, sólo dos estudios han descrito la reducción de costos operativos en la clínica atribuibles directamente al entrenamiento simulado (en el área de medicina intensiva y traumatología). ${ }^{18,19}$ Estudios de costo-efectividad aplicados a sistemas de salud locales podrían entregarnos herramientas valiosas respecto al impacto económico de la simulación. De esa manera, se podría sustentar una inversión razonable y ética en educación médica basada en simulación.

\section{CONCLUSIONES}

La simulación ha adquirido relevancia dentro de los programas de formación quirúrgica. A la fecha, las instancias donde entrenar destrezas esenciales para el desempeño clínico en un ambiente protegido representan momentos aislados dentro de los currículos. El establecimiento de un gimnasio de simulación para el desarrollo y mantenimiento 
de habilidades quirúrgicas es una opción factible y atractiva para residentes y cirujanos.

\section{Agradecimientos}

Agradecemos sinceramente a nuestra directora, la Dra. Marcia Corvetto, y al equipo administrativo y técnico que hace posible a diario el funcionamiento de este gimnasio: Marión Berrios, Andrés Campos, Raúl Nalvae y Francisco Serrano.

\section{REFERENCIAS}

1. McKenna DT, Mattar SG. What is wrong with the training of general surgery? Adv Surg. 2014; 48: 201210. doi: 10.1016/j.yasu.2014.05.010.

2. Richard K, Reznick HM. Changes in the wind. N Engl J Med. 2006; 355: 2664-2669. doi: 10.1056/ NEJMra054785.

3. Grantcharov TP, Reznick RK. Training tomorrow's surgeons: What are we looking for and how can we achieve it? ANZ J Surg. 2009; 79: 104-107. doi: 10.1111/j.1445-2197.2008.04823.x.

4. Choy I, Okrainec A. Simulation in surgery: perfecting the practice. Surg Clin North Am. 2010; 90: 457-473. doi: 10.1016/j.suc.2010.02.011

5. Hafford ML, Van Sickle KR, Willis RE, Wilson TD, Gugliuzza K, Brown KM, et al. Ensuring competency: Are fundamentals of laparoscopic surgery training and certification necessary for practicing surgeons and operating room personnel? Surg Endosc. 2013; 27: 118-126. doi: 10.1007/s00464-012-2437-7.

6. Darzi A, Munz Y. The impact of minimally invasive surgical techniques. Annu Rev Med. 2004; 55: 223 237. doi: 10.1146/annurev.med.55.091902.105248

7. Moulton CA, Dubrowski A, Macrae H, Graham B, Grober E, Reznick R. Teaching surgical skills: what kind of practice makes perfect?: a randomized, controlled trial. Am Surg. 2006; 244: 400-409. doi: 10.1097/01. sla.0000234808.85789.6a.

8. Agha RA, Fowler AJ. The role and validity of surgical simulation. Int Surg. 2015; 100: 350-357. doi: 10.9738/INTSURG-D-14-00004.1

9. Zendejas B, Cook DA, Bingener J, Huebner M, Dunn WF, Sarr MG, et al. Simulation-based mastery learning improves patient outcomes in laparoscopic inguinal hernia repair: a randomized controlled trial. Ann Surg. 2011; 254: 502-511. doi: 10.1097/SLA.0b013e31822c6994.

10. Scott DJ, Dunnington GL. The new ACS/APDS skills curriculum: Moving the learning curve out of the operating room. J Gastrointest Surg. 2008; 12: 213221. doi: $10.1007 / \mathrm{s} 11605-007-0357-y$.
11. Silverman E, Tucker SA, Imsdahl S, Charles JA, Stellato MA, Wagner MD, et al. Conducting elite performance training. Surg Clin North Am. 2015; 95: 839-854. doi: 10.1016/j.suc.2015.04.011.

12. Ericsson KA. Deliberate practice and the acquisition and maintenance of expert performance in medicine and related domains. Acad Med. 2004; 79: S70-S81. doi: 10.1097/00001888-200410001-00022.

13. Boyle E, Al-Akash M, Gallagher AG, Traynor O, Hill ADK, Neary PC. Optimising surgical training: use of feedback to reduce errors during a simulated surgical procedure. Postgrad Med J. 2011; 87: 524-528. doi: 10.1136/pgmj.2010.109363

14. Achurra P, Lagos A, Avila R, Tejos R, Buckel E, Alvarado $\mathrm{J}$, et al. Allowing new opportunities in advanced laparoscopy training using a full high-definition training box. Surg Innov. 2017; 24: 66-71. doi: $10.1177 / 1553350616672963$.

15. Hirsch EDJ. The core knowledge curriculum-what's behind its success? Educational Leadership. 1993; 50: 23-25, 27-30. [EJ 462 453].

16. Varas J, Mejía R, Riquelme A, Maluenda F, Buckel E, Salinas J, et al. Significant transfer of surgical skills obtained with an advanced laparoscopic training program to a laparoscopic jejunojejunostomy in a live porcine model: Feasibility of learning advanced laparoscopy in a general surgery residency. Surg Endosc. 2012; 26: 3486-3494. doi: 10.1007/s00464-012-2391-4.

17. Inzunza AM, Quezada GJ, Jarry TC, Torres GA, Tejos $S R$, et al. Impacto del primer "learning center" de cirugía mínimamente invasiva en Chile. Rev Cirugía. 2018; 71: [Consultado: 20 feb. 2019]. Disponible en: https://www.revistacirugia.cl/index.php/revistacirugia/ article/view/365

18. Bae DS, Lynch H, Jamieson K, Yu-Moe CW, Roussin C. Improved safety and cost savings from reductions in cast-saw burns after simulation-based education for orthopaedic surgery residents. J Bone Joint Surg Am. 2017; 99: e94. doi: 10.2106/JBJS.17.00199.

19. Cohen ER, Feinglass J, Barsuk JH, Barnard C, O'Donnell A, McGaghie WC, et al. Cost savings from reduced catheter-related bloodstream infection after simulation-based education for residents in a medical intensive care unit. Simul Healthc. 2010; 5: 98-102. doi: 10.1097/SIH.0b013e3181bc8304.

Correspondencia:

Dr. Julián Varas C

Marcoleta 377, Santiago de Chile.

Tel: +56223546483

E-mail: jevaras@uc.cl 